\title{
EL PLURIGRADO: UNA PRÁCTICA PEDAGÓGICA SINGULAR. ARGENTINA
}

\author{
Gabriela A. Fairstein \\ Adriana Pedernera ${ }^{2}$
}

\section{Resumen}

Este artículo se inscribe en el marco de un proyecto de investigación referido a las concepciones del proceso de enseñanza y de aprendizaje de los docentes de escuelas rurales y comprende tres apartados que intentan dar cuenta sobre sus aspectos más relevantes. En primer lugar, se presentan algunas notas relativas al diseño del proyecto; también se fundamenta la relevancia que asume la educación rural como temática que se constituye en objeto de nuestra investigación. A continuación, se describen las características que adopta el mencionado diseño en relación con el abordaje metodológico elaborado e implementado en dos escuelas rurales de la provincia de
La Rioja. Finalmente, se intenta caracterizar el aula plurigrado como una práctica pedagógica diferenciada del contexto de la escuela urbana.

Palabras claves: escuela rural, enseñanza, aprendizaje y aula plurigrado.

\begin{abstract}
This articles is inserted in the framework of a research project referred to the conceptions of the teaching and learning process of teachers of rural schools and it comprises three sections which intend to show its most relevant aspects. The first section, some notes related to the design of the project are presented, and also, the relevance that rural education adopts
\end{abstract}

\footnotetext{
Lịcenciada en Ciencias de la Educación. Universidad de Buenos Aires. Magister en Pedagogía Aplicada. Universidad Autónoma de Barcelona. Actualmente realiza el Doctorado en Pedagogia, en la Universidad Autónoma de Barcelona. Consultora Senior en el Programa de Naciones Unidas para el Desarrollo y realiza actividades de consultoria externa para organismos públicos.gfair@flacso.org.ar/gabifair@yahoo.com.ar

2 Licenciada en Psicopedagogia de la Universidad Nacional de La Rioja. Profesora en Psicopedagogia, titulo otorgado por esta misma Universidad. Diplomado Superior en Ciencias Sociales con mención en Constructivismo y Educación, otorgado por la Facultad Latinoamericana de Ciencias Sociales, Argentina. Jefa del Departamento de Investigación, Promoción y Desarrollo Educativo e integrante del Equipo de Gestión del Instituto de Formación Docente Continua “Alberto Cavero", Patquia, La Rioja. adrianapedernera@infovia.com.ar Recibido: 20 de octubre-2006 - Aprobado: 15 de noviembre-2006
} 
as the subject matter which constitutes our object of research. In the following lines, some characteristics adopted by such design in relation to the methodological approach developed and implemented in two rural schools of the province of La Rioja are described. Finally, this article attempts to characterize the multi-grade classroom as a pedagogical practice different from the context of the urban school.

Keywords: rural school, teaching, learning, multi-grade classroom.

\section{Algunas notas sobre el proyecto de investigación}

Q objetivo general de la investigación consiste en caracterizar las concepciones acerca del proceso de enseñanza y aprendizaje de docentes de escuelas rurales, para comprenderlas e inferir los supuestos, con el propósito de ayudar a los docentes en formación a anticipar aspectos de la práctica en el aula plurigrado y la escuela rural. En concreto, nuestro problema de investigación se traduce en el siguiente interrogante: ¿cuáles son las concepciones de enseñanza y aprendizaje de los docentes de escuelas ubicadas en zonas rurales?

A partir de esta pregunta general, nos interesará indagar sobre estas concepciones para identificar teorías psicológicas y didácticas, así como supuestos epistemológicos, que los y las docentes movilizan a la hora de su práctica pedagógica. Por otro lado, y dado que la investigación se centra en diversos aspectos del quehacer pedagógico -discurso, planificaciones, práctica áulica-, pretendemos detectar puntos de coherencia y de contradicción en el seno de estas concepciones.

La temática de nuestra investiga ción se fundamenta en el hecho de que el Instituto de Formación Docente, del cual surge el proyecto de investigación ${ }^{3}$, tiene como campo de formación focalizada, la educación rural, como eje transversal en la formación que reciben los y las estudiantes desde el $1^{\circ}$ al $3^{\circ}$ año de la carrera. Esto implica que egresan como docentes de la educación general básica para $1^{\circ}$ y $2^{\circ}$ ciclos con una formación específica en educación rural. Por ello, consideramos que la temática abordada en el presente trabajo enriquecerá los conocimientos existentes en el campo de formación focalizada, habida cuenta de que el material bibliográfico y las producciones teóricas elaboradas sobre la educación en el medio rural son insuficientes y dispersos. En este sentido, expresa Beatriz Fainholc, "La educación rural, a pesar de los numerosos proyectos, propuestas e intenciones, sólo representó una preocupación

3 Instituto ubicado en la localidad de Patquia del Departamento Independencia. 
pequeña o marginal del sector público" (1992, p. 9). Asimismo, el Instituto Internacional de Planeamiento de la Educación de la UNESCO menciona:

"En la actualidad, el 30\% de las aulas del mundo son del tipo multigrado. Sin embargo [...] las autoridades gubernamentales suelen ignorar estas escuelas en tanto que, pese a su creciente número y los resultados obtenidos, rara vez forman parte de las estadísticas e iniciativas de investigación educativa" (2004, p. 7).

En concordancia con estos datos, cabe señalar que en el territorio de la provincia de La Rioja, el 75\% de escuelas de educación general básica corresponde a contextos rurales, según un estudio realizado por la Unidad de Estadística Educativa de la Dirección General de Planeamiento, dependiente del Ministerio de Educación de la Provincia en el año 2002. Este dato cuantitativo pone de manifiesto una realidad educativa que, desde nuestra mirada, es significativa y relevante en comparación con la escasa producción de material de estudio especializado.

Por otra parte, nuestro interés por las concepciones de los docentes acerca de los procesos de enseñanza y aprendizaje deriva de la consideración de que las prácticas pedagógicas se apoyan, implícita o explícitamente, en supuestos particulares acerca del conocimiento, de la enseñanza, del aprendizaje y del sujeto que aprende. Estos supuestos inciden en las estrategias de enseñanza que el o la docente despliega en el contexto áulico y en las estrategias de aprendizaje que estimula en el o la estudiante. En este sentido, diversos autores coinciden en destacar la importancia que tiene para el proceso educativo, el analizar las ideas que subyacen en el accionar de todo docente:

"Los profesores mantienen determinadas concepciones sobre la educación y la enseñanza. Unas son más racionales y explícitas...Otras son menos articuladas, con escasa coherencia lógica y con menor consistencia entre las diversas ideas que las componen...A pesar de no tener perfiles claros y escapar a la toma de conciencia inmediata, operan con enorme fuerza en la explicación de los hechos y en la orientación del comportamiento" (Marchesi y Martín, 1998, p. 286). 
Asimismo, Carretero menciona:

"Si se pretende mejorar el proceso de enseñanza y aprendizaje es preciso que los docentes podamos conocer las teorías que subyacen muchas veces a nuestra práctica y de esa manera facilitar un posible proceso de cambio. Incluso en el caso de que las teorías que tenemos no sean las causas fundamentales de nuestro comportamiento, sino más bien una justificación a posteriori, el conocimiento detallado de dichas teorías sigue siendo un asunto de central importancia ya que cumplen una función en el complicado engranaje de las relaciones entre pensamiento y acción" (1998, p. 17).

\section{Breve descripción del diseño metodológico}

El abordaje metodológico utilizado está enmarcado dentro de una perspectiva cualitativa-interpretativa, por cuanto se pretende la comprensión del fenómeno y no la comprobación. A partir del análisis del discurso y del quehacer educativo cotidiano de los propios actores involucrados en el proceso, intentamos acercamos a la realidad que se constituye en nuestro objeto de indagación. Así, el diseño no parte de hipótesis previas a confirmar, sino de un conjunto de preguntas e interrogantes que ayudaron a delimitar el problema y guiaron la búsqueda y el análisis de los datos obtenidos.

Desde el punto de vista del tipo de los datos que se han de recoger para llevar a cabo el proyecto de investigación, el diseño utilizado se enmarca dentro de los diseños de campo, por cuanto los datos son de primera mano (Sabino, 1996). Considerando que nuestro objeto de estudio -las concepciones de los docentes- se manifiesta en diversos planos y momentos del quehacer pedagógico, se privilegiaron como fuentes de información las siguientes: el discurso del docente acerca de su práctica, el análisis de las planificaciones y la observación de la práctica áulica. Entendemos que las concepciones de enseñanza y aprendizaje se cristalizan y actualizan de diferentes maneras en cada uno de estos planos de la tarea docente. En función de ello, la consideración conjunta de los datos de estas fuentes en la etapa de análisis nos permitirá reconstruir, de un modo lo más acabado posible, el contenido de dichas concepciones a través de una estrategia de triangulación.

En relación con los instrumentos utilizados para la recolección de datos, estos se relacionan con cada una de las fuentes de información y corresponden tanto a las tradiciones cualitativas como cuantitativas de la metodología 
de investigación. Consideramos que la combinación de instrumentos provenientes de ambas perspectivas resulta compatible en nuestro diseño, en tanto nos permite obtener información diversa para una comprensión integral más acabada del objeto de indagación. Los instrumentos de recolección de datos utilizados fueron:

- Entrevistas individuales a docentes.

- Encuestas semiestructuradas a docentes.

- Registros de clase (observación no participante).

- Consulta de documentación escolar:

- Proyecto educativo institucional.

- Planificaciones didácticas (anual y periódica).

Por último, cabe señalar que hemos seleccionado el muestreo no probabilístico y dentro de este, la muestra intencional. La selección de la muestra fue el resultado de una tarea previa de conocimiento del universo y estuvo orientada hacia la selección de escuelas que pudieran considerarse representativas de dicho universo. En función de ello, se han tomado dos unidades de análisis:

- Una escuela ubicada a $92 \mathrm{~km}$ de la localidad de Patquía (cabecera del Departamento Independencia de la provincia de La Rioja), contando con nivel inicial y educación general básica, $1^{\circ}, 2^{\circ}$ y $3^{\circ}$ ciclos. Su planta funcional comprende cuatro docentes, un docente para nivel inicial, un docente para $1^{\circ}$ ciclo, un docente para $2^{\circ}$ ciclo y un docente para $3^{\circ}$ ciclo.

- Una escuela ubicada a $28 \mathrm{~km}$ de Patquía, contando con educación general básica, $1^{\circ}, 2^{\circ}$ y $3^{\circ}$ ciclos. Su planta funcional comprende dos docentes, un docente para $1^{\circ}$ y $2^{\circ}$ ciclos y un docente para $3^{\circ}$ ciclo.

\section{Hacia algunos resultados provisorios: caracterizando el aula plurigrado}

Si bien nos encontramos aún inmersos en el análisis e interpretación de los insumos obtenidos durante el trabajo de campo realizado y en la triangulación de estos, podemos llegar a algunos resultados provisorios. Nuestra inserción en las escuelas ubicadas en zonas rurales del Departamento Independencia nos permitió acceder al conocimiento del mundo rural, como un ámbito con características sociales, culturales y económicas peculiares, asumiendo la escuela un rol central y protagónico. 
Si bien las dos escuelas analizadas conservan su propia identidad y singularidad, comparten, en el ámbito organizativo y pedagógico, un rasgo central: el trabajo con grados simultáneos. Esta configuración pedagógicodidáctica, denominada plurigrado o multigrado, implica que los procesos de enseñanza y de aprendizaje que se desenvuelven en ellas presenten un carácter heterogéneo y multidimensional, advirtiéndose una pluralidad de criterios metodológicos y organizativos en función de la realidad particular de cada una de ellas.

Para comprender qué se entiende por plurigrado, cabe señalar que este se constituye en un ámbito de aprendizaje en un doble sentido: para el o la estudiante (niño o niña que vive en zonas rurales) supone la única posibilidad y oportunidad brindadas por el Estado en el marco de los aprendizajes correspondientes a la educación sistemática; para el o la docente implica el desafio cotidiano (aprendizaje diario) de enfrentar una modalidad organizativa y pedagógica de la escuela para la cual, la mayoría de las veces, no está formado ni capacitado. Al ser diferente a la modalidad existente en la escuela urbana, modelo a partir del cual suele organizarse la formación y capacitación, el plurigrado exige al docente un replanteo diferenciado de su práctica pedagógica. Es decir, si bien todo docente se sujeta a una revisión constante de su accionar, la realidad rural lo enfrenta con la necesidad de buscar propuestas pedagógico-didácticas diferenciadas, que le permitan superar la complejidad que asumen los componentes de su práctica pedagógica. Así, la búsqueda y el ensayo de diferentes modalidades de agrupamientos y metodologías de enseñanza, de modalidades de aprendizaje y formas de evaluación, son una constante en la educación rural y para las cuales el docente se encuentra en soledad en la mayoría de los casos.

Según la literatura especializada existente sobre el tema, la modalidad pedagógica del plurigrado puede caracterizarse por las siguientes notas:

- Constituye la forma o modalidad organizativa que adoptan las escuelas en contextos rurales.

- Incluye una diversidad de situaciones que responden a la característica de que comparten estudiantes de diferentes años de escolaridad, un mismo espacio y tiempo.

- Constituye una respuesta administrativa más que pedagógica de los sistemas educativos para atender a la demanda rural. 
En este sentido, expresa Justa Ezpeleta:

"Como en otros países de la región, esta fue la manera de responder a las particularidades de la demanda rural, sin atender a la naturaleza de la acción pedagógica que ella requería. La estructura conocida y disponible de escuela, nacida para medios urbanos, se consideró pertinente para ser aplicada al medio rural con escasa y heterogénea población infantil. El multigrado, más que respuesta y estrategia pedagógica, en este caso fue el resultado de una operación administrativa que, para brindar el servicio, encontró la solución de reducir el personal en concordancia con la cantidad de alumnos. La enseñanza, los contenidos curriculares y las obligaciones administrativas quedaron sometidos a las regulaciones propias de otra realidad escolar, la urbana, con un maestro por grado" (1997, p. 3).

Desde nuestra perspectiva, el plurigrado constituye una práctica pedagógica compleja, por cuanto el proceso que esta implica, desarrollado en el contexto del aula y centrado en el enseñar y el aprender, involucra diversos aspectos (agrupamientos de los y las estudiantes, estrategias de enseñanza, tratamiento del contenido, actividades de aprendizaje, intervenciones del docente, participación del alumno, recursos didácticos, actividades de evaluación), que se encuentran y entrecruzan en un mismo tiempo y espacio común (sala de clase) dirigidos hacia estudiantes de diferentes edades evolutivas (en su desarrollo cognitivo, psicomotriz y social) y de diferentes grados o años según la escolaridad: esto producirá ciertos espacios y tiempos de intersección o convergencia de los momentos de la clase (inicio, desarrollo y cierre) durante el despliegue realizado por el docente en el transcurso de esta. Además, dicha práctica pedagógica se encuentra atravesada por factores socioeconómicos y culturales de zonas desfavorables, enmarcándose en un contexto de aislamiento y soledad, pero que también supone amplios márgenes de libertad.

Si bien se trata de un análisis provisional, podemos distinguir las siguientes características específicas del aula plurigrado:

- Práctica pedagógica implementada en el contexto áulico para diferentes grados o años de uno o más ciclos, de manera simultánea en todos los aspectos o dimensiones que esta implica, lo que constituye una modalidad pedagógica singular, cuya complejidad y características particulares la diferencian cualitativamente del aula de la escuela urbana. 
La variabilidad que presentan las estrategias de inicio, de desarrollo y de cierre, considerando los momentos de la clase o la enseñanza para cada grado. Dichas estrategias pueden ser presentadas de manera simultánea o alternadas para los diferentes grados (en función del tiempo destinado para cada espacio curricular). $\mathrm{O}$ bien, presentadas de manera unificada o diferenciada para los diferentes grados (en función de los agrupamientos realizados por el docente). Tales agrupamientos pueden responder a diversos criterios establecidos por el o la docente -por grado, por grados próximos, por temas, por niveles-, o bien, constituir todos los estudiantes un solo grupo.

- La presencia y escucha por parte de los alumnos que comparten el aula de conocimientos que le fueron enseñados (no sabemos si aprendidos) en años anteriores y de conocimientos correspondientes a años escolares posteriores al que se encuentra transitando en un determinado año escolar. Esta condición, que se presenta como impuesta para el proceso de aprendizaje de cada estudiante, por cuanto es ineludible e inherente a dicho contexto escolar, asume dos cualidades: la de favorecer o la de obstaculizar el proceso de aprendizaje del sujeto. El signo que tome dependerá de: a) el modo que el docente interprete la incidencia de esta condición en el proceso de aprendizaje de cada estudiante y, por ende, b) el modo que el docente aborde, encare o trabaje dicha condición -desde la enseñanza- durante el trabajo áulico cotidiano.

- El tiempo dedicado al tratamiento de los contenidos de un determinado espacio curricular no está determinado por los límites convencionales - marcados habitualmente por la campana- y establecidos desde la organización escolar para delimitar la duración de la hora de clase y del recreo, sino quie queda libre a la decisión que adopte el docente en cada jornada escolar. Ello supone un corte deliberado por parte del docente en el que, por un lado, se pone de manifiesto la autonomía y flexibilidad de que dispone, para manejar los tiempos de la enseñanza y el momento en que se interrumpe la secuencia didáctica, lo que incidirá en el proceso de aprendizaje de cada alumno. Pero además, evidencia la concepción que posee el o la docente no sólo sobre el qué, cómo y cuándo enseñar, sino también sobre el cuándo dejar de enseñar, en tanto es quien produce los cortes (no impuestos desde afuera) en el proceso de enseñanza. Al igual que en el caso anterior, el signo que adopte este rasgo, es decir, si los cortes resultan adecuados o no, dependerá del modo en que el docente conciba el papel que cada secuencia didáctica 
puede tener para favorecer los procesos cognitivos de sus estudiantes.

- Tendencia predominante en el docente a dirigir una atención más personalizada hacia cada estudiante en particular, lo que no significa necesariamente enseñanza individualizada. Si bien la atención personalizada se vería favorecida en las escuelas de estos contextos por la poca población estudiantil, y aparece como una necesidad en función de la heterogeneidad que caracteriza al grupo de estudiantes, y las condiciones sociales, culturales, económicas y geográficas en las que se encuentra inmersa la escuela, esta dependerá de la decisión de cada centro educativo y de cada docente.

- La movilización permanente de los esquemas y del conocimiento práctico que posee el docente para dar respuestas a las demandas de sus estudiantes de diversos grados, ciclos y niveles, de manera simultánea e inmediata, lleva a que el o la docente se encuentre en un estado dialéctico constante entre el "saber" y el "saber hacer". Asimismo, supone un elevado nivel de apertura y disposición para resolver todo aquello que no estaba previsto, proveniente desde los múltiples grados simultáneos. Esta característica del plurigrado interpela la práctica pedagógica del docente de manera sistemática e ineludible.

En conclusión, podemos considerar, entonces, que el plurigrado constituye una forma particular de abordar la práctica pedagógica que se presenta al docente como condición y exigencia profesional para ejercer su tarea en escuelas de contextos rurales, configurando un espacio privilegiado y potenciado para ejercer permanentemente la toma de decisiones en relación con los diversos aspectos que supone el enseñar y el aprender.

Estas decisiones varian de un docente a otro, según diversos factores, entre los que cabe mencionar: la teoría didáctica que haya adoptado; su modo de entender la práctica pedagógica; sus concepciones acerca de la enseñanza, el aprendizaje, el docente y el alumno; la experiencia que posea en escuelas de contextos rurales; su particular modo de enfrentar los factores sociales, económicos y culturales propios de zonas desfavorables; su grado de apertura, disponibilidad y flexibilidad para entender, actuar y reflexionar sobre dicha práctica.

Para finalizar, deseamos destacar que con la lectura, análisis e interpretación de los datos y la triangulación de estos, pretendemos llegar a conclusiones que nos permitan contribuir en la búsqueda de nuevos conocimientos centrados en la educación rural de nuestro medio. 


\section{REFERENCIAS}

Carretero, M. y otros. (1998). Procesos de enseñanza y aprendizaje. Buenos Aires: Aique.

Ezpeleta, J. (1997). “Algunos desafíos para la gestión de las escuelas multigrado". En Revista Iberoamericana de Educación, $\mathrm{N}^{\circ} 15$ (septiembre-diciembre), p. 3.

Fainholc, B. (1992). Educación rural: temas claves. Buenos Aires: Aique.

Marchesi, A. y Martín, E. (1998). Calidad de la enseñanza en tiempos de cambio. Madrid: Editorial Alianza.

Ministerio de Educación, Ciencia y Tecnología de la Nación (1999). "El sujeto del aprendizaje escolar como sujeto colectivo". En Proyecto Tercer Ciclo de la $E G B$ en Escuelas Rurales. Plan Social Educativo. Buenos Aires: MECyT.

Ministerio de Educación, Ciencia y Tecnología de la Nación. (2004). "Ejemplos de situaciones de enseñanza en plurigrado". Informe de la Dirección Nacional de Gestión Curricular y Formación Docente, Área de Educación Rural. Buenos Aires: MECyT.

Ministerio de Educación de la Provincia de La Rioja. (2002). Relevamiento Anual. Unidad de Estadística Educativa, Dirección General de Planeamiento. La Rioja: ME.

Sabino, C. (1996). El proceso de investigación. Buenos Aires: Lumen-Humanitas.

UNESCO. (2004). "Escuelas multigrado: una solución rural". Educación Hoy (9), Boletín del sector educación de la UNESCO. 